\title{
AVALIAÇÃO DE INDICADORES OPERACIONAIS DE UM SERVIÇO DE ATENDIMENTO MÓVEL DE URGÊNCIA*
}

\author{
Naldiana Cerqueira Silva ${ }^{1}$, Lidya Tolstenko Nogueira ${ }^{2}$
}

RESUMO: O atendimento pré-hospitalar se consolidou no Brasil a partir das normas estabelecidas em portarias ministeriais que culminaram com a implantação de Serviços de Atendimento Móvel de Urgência no país. Neste estudo, realizou-se a avaliação dos resultados produzidos por este Serviço em Teresina- Piauí, analisando a natureza das ocorrências, o tempo resposta e referenciamento dos atendimentos no período de 2005 a 2011. Trata-se de pesquisa avaliativa, descritiva, quantitativa com dados obtidos a partir das fichas de atendimento e consolidação. Verificou-se maior número de solicitações para urgências clínicas em adultos, demanda elevada e decrescente para urgências clínicas e obstétricas, e ascendente para acidentes de trânsito. Constatou-se que o tempo de deslocamento até o local de atendimento foi maior que o recomendado para a zonas urbana e rural. Conclui-se que no município do estudo este Serviço necessita reduzir o tempo resposta, com otimização de oferta hospitalar. PALAVRAS-CHAVE: Socorro de urgência; Assistência pré-hospitalar; Avaliação de programas e projetos de saúde.

\section{EVALUATION OF OPERATIONAL INDICATORS IN A MOBILE EMERGENCY CARE SERVICE}

\begin{abstract}
Pre-hospital attendance has been consolidated in Brazil based on norms established in ministerial ordinances which culminated in the implantation of Mobile Emergency Care Services in the country. This study evaluated the results produced in Teresina in the state of Piauí, analyzing the nature of the occurrences, the response time, and the referral of the attendances in the period 2005 -2011. It is evaluative, descriptive and quantitative research, based on data obtained from patient notes and consolidation of the same. A higher number of requests for clinical emergencies was ascertained in adults, a high but shrinking demand for clinical and obstetric emergencies, and an increasing demand due to traffic accidents. It was determined that the time spent travelling to the care center was greater than recommended for urban or rural zones. It is concluded that in the municipality under study, this Service needs to reduce the response times, with optimization of the provision of hospital care. KEYWORDS: Emergency help; Pre-hospital care; Evaluation of health programs and projects.

\section{EVALUACIÓN DE INDICADORES OPERACIONALES DE UN SERVICIO DE ATENDIMIENTO MÓVIL DE URGENCIA}

\begin{abstract}
RESUMEN: El atendimiento prehospitalar se ha consolidado en Brasil a causa de las normas establecidas en portarías ministeriales que culminaron con la implantación de Servicios de Atendimiento Móvil de Urgencia en el país. En este estudio, se realizó evaluación de los resultados producidos por este servicio en Teresina, Piauí, analizando la naturaleza de las ocurrencias, el tiempo de respuesta y el referencial de los atendimientos en el periodo de 2005 a 2011. Es una investigación evaluativa, descriptiva, cuantitativa con datos obtenidos por las fichas de atendimiento y consolidación. Se ha verificado mayor número de solicitaciones para urgencias clínicas en adultos, demanda elevada y decreciente para urgencias clínicas y obstétricas, y ascendiente para accidentes de tránsito. Se verificó que el tiempo de desplazamiento hasta el local de atendimiento fue mayor que lo recomendado para zonas urbana y rural. Se concluye que en el municipio del estudio este servicio necesita reducir el tiempo de respuesta, con optimización de oferta hospitalar. PALABRAS CLAVES: Socorro de urgencia; Asistencia prehospitalar; Evaluación de programas y proyectos de salud.
\end{abstract}

*Extraído da dissertação de Mestrado intitulada Avaliação Normativa do SAMU Teresina, apresentado ao Programa de Mestrado em Enfermagem da Universidade Federal do Piauí - UFPI, 2011.

${ }^{1}$ Enfermeira do Serviço de Atendimento Móvel de Urgência - SAMU de Teresina - PI. Mestre em Enfermagem. Professora da Universidade Estadual do Piauí e da Faculdade Integral Diferencial.

${ }^{2}$ Enfermeira. Doutora em Enfermagem. Professora Associada do Departamento de Enfermagem e do Programa de Mestrado em Enfermagem da UFPI. 


\section{INTRODUÇÃO}

Segundo o Ministério da Saúde, a urgência e a emergência no Brasil são percebidas como setores deficientes do sistema de saúde e se configuram como áreas problemáticas do Sistema Único de Saúde, no qual as diretrizes de descentralização, regionalização e hierarquização estão pouco implementadas ${ }^{(1)}$. Nesse paradigma, surgiu a Política Nacional de Atenção às Urgências e, consequentemente, o Serviço de Atendimento Móvel de Urgência (SAMU), com o intuito de induzir a organização da rede de atenção e estruturação dos serviços e constituiu importante observatório do sistema de saúde brasileiro, sendo imprescindível a discussão e a análise com a finalidade de se propor soluções frente à problemática.

O Atendimento Pré-hospitalar (APH) é caracterizado como toda assistência prestada fora do ambiente hospitalar, de forma direta ou indireta, afim de oferecer uma resposta apropriada. Com a implantação do SAMU para a consolidação do APH, adveio a regulação médica. Também os fluxos de solicitações de atendimento passaram a ser operacionalizados com a garantia de acesso equânime à rede de saúde de cada município, tendo em vista que o SAMU opera com o princípio de vaga zero, o que implica o atendimento independente da existência, ou não, de leitos vagos nos hospitais. Sua atuação compreende desde uma orientação até o envio de uma viatura de suporte básico, ou avançado, ao local da ocorrência, conforme solicitação do usuário e gravidade do caso ${ }^{(2)}$.

O SAMU é importante para o sistema de saúde vigente, pois induz a organização da rede de atenção e proporciona a estruturação dos serviços de saúde ${ }^{(3)}$. Dessa forma, o SAMU do Município de Teresina, Piauí, como os demais, é avaliado pelo Ministério da Saúde, que colhe, mensalmente, dados relativos aos atendimentos para avaliação e monitoramento. Avaliar significa conferir valor, manifestar-se em relação a alguma coisa, fundamentado em um juízo, utilizando um método específico ${ }^{(4)}$, enquanto que a avaliação de resultado se destina a analisar a produção do serviço prestado, ou seja, avaliar as ações que se presta à população ${ }^{(5)}$.

Focando a discussão sobre o processo avaliativo deve-se ressaltar que há relação histórica entre o desenvolvimento da avaliação como campo de investigação científica e o desenvolvimento das políticas públicas e de saúde, com o objetivo de reduzir a pobreza e promover o desenvolvimento social. Nesse contexto, surgiu o conceito de avaliação dos programas públicos ${ }^{(6)}$ que, de uma forma geral, são instituídos com o propósito de servir à população para qual se destinam. Assim, esses programas e serviços devem ser objeto de monitoramento e avaliação, com a finalidade de verificar se os objetivos de implantação estão sendo atingidos.

Elegeu-se como objetivo avaliar os indicadores operacionais obtidos quanto à natureza das ocorrências, o tempo resposta e o referenciamento dos atendimentos produzidos pelo SAMU de Teresina, Piauí. Pretendeu-se discutir a avaliação do serviço, com a justificativa da inexistência de estudo com essa abordagem, para o fortalecimento de políticas avaliativas na área, buscando visibilidade ao trabalho desenvolvido. O presente estudo oferece subsídios aos profissionais de saúde, gestores e população para que possam conhecer os indicadores operacionais, valorizar e contribuir para o crescimento e o aprimoramento do SAMU, como serviço indispensável à sociedade.

\section{MÉTODO}

Trata-se de uma pesquisa avaliativa, descritiva, com abordagem quantitativa que abrangeu informações pertinentes aos resultados obtidos pelo referido SAMU, no período de janeiro de 2005 a dezembro de 2011. Este serviço atende a cidadãos acometidos por agravos à saúde, de natureza clínica, psiquiátrica, cirúrgica, traumática, obstétrica e ginecológica, com acesso telefônico gratuito pelo número nacional 192, nas regiões urbana e rural do município. Possui duas viaturas de transporte, 10 de Suporte Básico de Vida e três de Suporte Avançado de Vida; conta com 171 profissionais que desenvolvem suas atividades em Unidades Móveis de Urgência, sendo 62 auxiliares e/ ou técnicos em enfermagem, 62 condutores de veículos de urgência, 17 enfermeiros e 30 médicos.

Foram consideradas como fontes de dados informações obtidas nas fichas de atendimento e nos consolidados do serviço. Examinamos os quantitativos que deram origem ao deslocamento das unidades móveis e os que geraram orientações pelos médicos reguladores, que corresponderam a 445.238 no período em estudo. Os dados coletados foram digitados duplamente no programa Microsoft Excel e, depois, importados para o programa Statistical Package for the Social Sciences - SPSS (versão 12.0 for Windows), no qual foram tabulados. Utilizou-se a estatística descritiva face a impossibilidade de uma análise mais aprofundada, sendo explorada por meio de técnica univariada, configurando-se em uma pesquisa de caráter estatístico descritivo. 
Para subsidiar a discussão dos dados e avaliação comparativa, procedeu-se levantamento de informações sobre o SAMU nas capitais brasileiras para identificar o que mais se assemelhava com a realidade de Teresina no que diz respeito à estrutura, contingente populacional $\mathrm{e}$ disponibilidade de informações para análise comparativa. Para proceder à avaliação da natureza dos atendimentos, optou-se pelo SAMU da região metropolitana de Florianópolis - Santa Catarina, por não haver disponibilidade de informações acessíveis nos sites dos serviços das Regiões Norte e Nordeste. Em relação ao tempo resposta e ao referenciamento, realizou-se a análise comparativa com artigos publicados em periódicos científicos.

O estudo atendeu as exigências da Resolução 196/96, do Conselho Nacional de Saúde, com aprovação pelo Comitê de Ética e Pesquisa da Universidade Federal do Piauí, com Certificado de Apresentação para Apreciação Ética 0209.0.045.000-09.

\section{RESULTADOS}

As ocorrências geradas para o SAMU variaram nas mais diversas causas, como mostra a tabela 1.

Constatou-se que no período do estudo o SAMU realizou 445.238 atendimentos, dos quais 295.726 geraram deslocamento das viaturas. O maior quantitativo de ocorrências completadas foi gerado por urgência clínica em adulto, seguido por remoções inter-hospitalares e urgências obstétricas. Os acidentes de trânsito ocuparam a terceira posição em número de ocorrências, os traumas por agressão física ocuparam a sétima colocação; salienta-se número significativo de falsos chamados.

A figura 1 mostra a evolução ao longo dos anos da natureza das ocorrências realizadas pelo SAMU do município de Teresina.

A Tabela 2 aponta a média, o índice de confiança, desvio padrão, mínima e máxima dos tempos de deslocamento no período, o tempo médio de resposta em chamados urbanos e rurais e o tempo médio de transporte decorrente dos atendimentos.

Pode-se observar que a média do tempo resposta do SAMU do Município de Teresina na zona urbana foi inferior à média do tempo resposta para zona rural, bem como o tempo médio de transporte nas duas zonas.

Realizou-se consolidado do referenciamento dos atendimentos produzidos pelo SAMU, de acordo com o tipo de estabelecimento de saúde aos quais os pacientes foram encaminhados, como demonstra a tabela 3 .

Os hospitais municipais de urgência e emergência de média e baixa complexidade receberam o maior número de pacientes, quando comparados aos hospitais de urgência e emergência de alta complexidade, referência para o estado. Os hospitais privados participam no recebimento dos pacientes que têm plano de saúde e/ou se propõem a assumir as despesas do tratamento. $\mathrm{O}$ termo "outros" se refere à média de atendimento inferior a 100 referenciamentos/ano, os quais foram encaminhados a hospitais públicos e privados não especificados na tabela e a clínicas e ambulatórios do município de Teresina.

Tabela 1- Média, mínima e máxima de atendimentos por natureza de ocorrência do SAMU do município de Teresina - PI, entre os anos de 2005 a 2011. Teresina, 2012

\begin{tabular}{lcccc}
\hline & Mín. - Máx.1 & Média & Total & \% \\
\hline Urgência clínica adulto & $10.943-20.573$ & 14.648 & 102.540 & 23,0 \\
Urgência obstétrica & $4.571-9.250$ & 6.779 & 47.457 & 10,1 \\
Trauma por acidente de trânsito & $2.728-5.546$ & 4.103 & 28.721 & 6,5 \\
Trauma por queda & $1.630-3.058$ & 2.172 & 15.280 & 3,4 \\
Urgência clínica pediátrica & $698-2.355$ & 1.656 & 11.592 & 2,7 \\
Urgência psiquiátrica & $1.246-1.759$ & 1.492 & 10.443 & 2,4 \\
Trauma por agressão física & $1.151-1.824$ & 1.382 & 9.687 & 2,3 \\
Falsos chamados (Trotes) & $175-665$ & 330 & 2.311 & 0,6 \\
Outros* & $176-668$ & 225 & 1.575 & 0,5 \\
Remoções inter-hospitalares & $6.618-10.242$ & 8.841 & 61.888 & 13,9 \\
Óbitos antes e durante o transporte & $557-646$ & 604 & 4.232 & 1,0 \\
Orientações & $17.841-23.139$ & 21.358 & 149.512 & 33,6 \\
(não geraram deslocamento) & & & & \\
\hline
\end{tabular}

* Corresponde à soma dos casos de afogamentos, choque elétrico, envenenamento e queimaduras.

Fonte: Fichas de Atendimento do SAMU - Teresina dos anos de 2005 a 2011. 


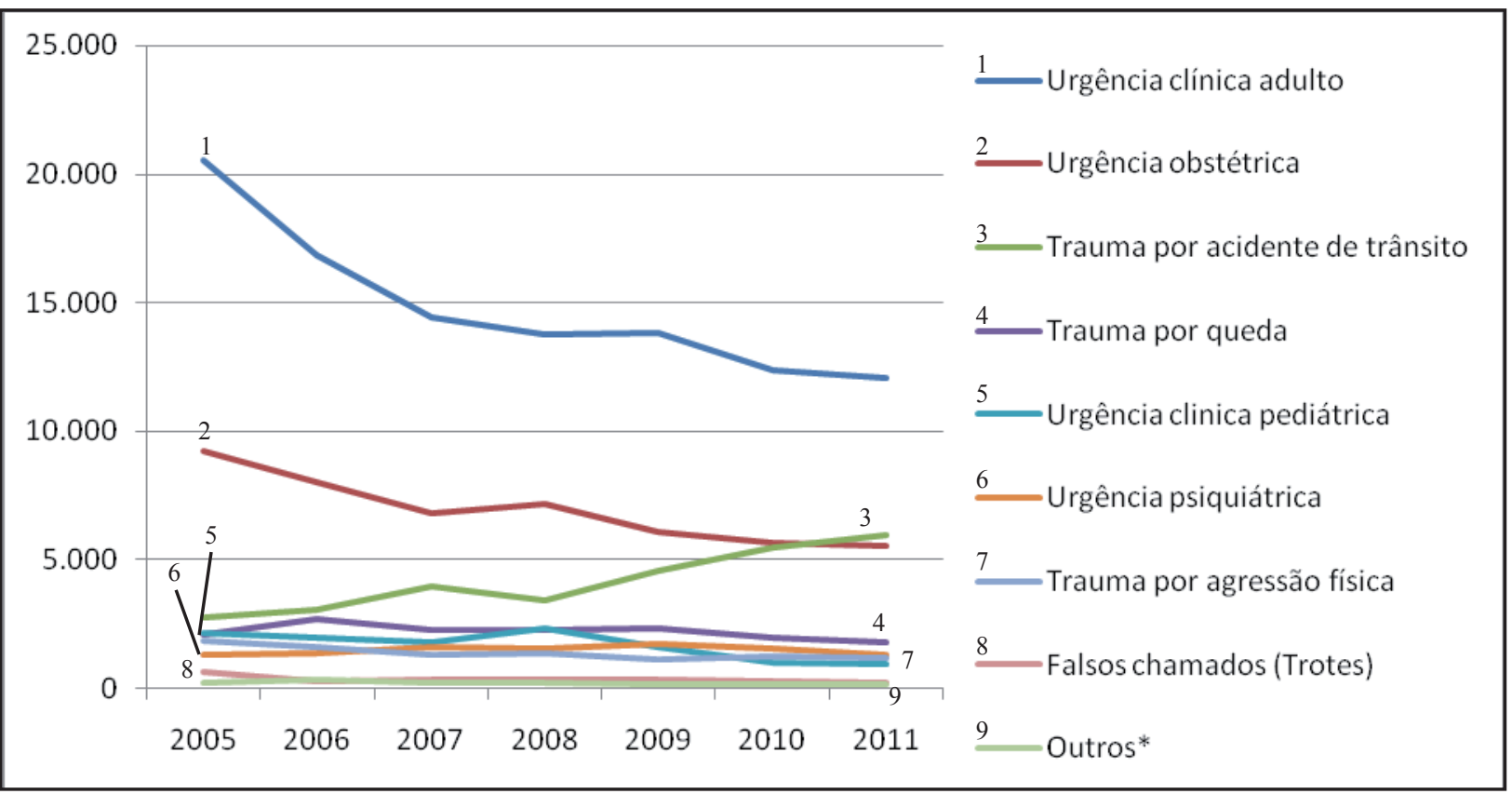

Figura 1 - Representação da natureza das ocorrências atendidas pelo SAMU Teresina - PI no período de 2005 a 2011. Fonte: Fichas de Atendimento do SAMU Teresina de 2005 a 2011.

Tabela 2 - Análise univariada do tempo de deslocamento das ambulâncias do SAMU do município de Teresina no período de 2005 a 2011. Teresina, 2012

\begin{tabular}{lcccc}
\hline & $\begin{array}{l}\text { Deslocamento até o } \\
\text { local do chamado ur- } \\
\text { bano/minuto }\end{array}$ & $\begin{array}{l}\text { Decal docamento até o } \\
\text { ral/minuto }\end{array}$ & $\begin{array}{l}\text { Deslocamento do lo- Deslocamento do lo- } \\
\text { cal urbano até o hos- cal rural até o hospi- } \\
\text { pital/minuto }\end{array}$ & $\begin{array}{l}\text { tal/minuto } \\
\text { tal }\end{array}$ \\
\hline Média & 13,6 & 32.8 & 11,3 & 28,3 \\
IC de 95\% & $12,4-14,7$ & $32,7-36,4$ & $10,1-12,2$ & $26,1-31,0$ \\
Desvio padrão & 0,8 & 1,5 & 0,8 & 1,9 \\
Mínima & 13 & 33 & 10 & 27 \\
Máxima & 15 & 37 & 12 & 32 \\
\hline
\end{tabular}

Fonte: Fichas de Atendimento do SAMU do município de Teresina de 2005 a 2011.

Tabela 3- Média, mínima e máxima do referenciamento do SAMU do Município de Teresina - PI, entre 2005 a 2011. Teresina, 2012

\begin{tabular}{lcc}
\hline & Média & Mínimo-Máximo \\
\hline Hospital de Urgência e Emergência referência para o estado & 9.403 & $5.002-13.458$ \\
Hospitais de Urgência e Emergência municipais(média e baixa complexidade) & 21.617 & $14.668-28.532$ \\
Maternidade de referência para o estado & 3.906 & $2.736-4.071$ \\
Maternidades Municipais & 4.739 & $3.428-4.808$ \\
Hospital psiquiátrico de referência para o estado & 1.547 & $1.279-1.777$ \\
Hospital de doenças tropicais referência para o estado & 612 & $514-623$ \\
Hospitais privados de Teresina & 1.702 & $960-2.399$ \\
Outros & $<100$ & - \\
\hline
\end{tabular}

Fonte: Fichas de Atendimento do SAMU do município de Teresina de 2005 a 2011. 


\section{DISCUSSÃO}

O SAMU da região metropolitana de Florianópolis, selecionado para a avaliação comparativa, presta atendimento a população aproximadamente $19 \%$ maior que a de Teresina e conta com 11 viaturas básicas e quatro viaturas avançadas. Aquele serviço consolida dados de forma semelhante aos de Terezina e os disponibiliza por meio eletrônico. De acordo com o relatório de 2010, da Secretaria Estadual de Saúde de Santa Catarina, Florianópolis faz seu consolidado anual de forma mais detalhada, incluindo as ocorrências por traumas e causas externas, que se subdividem em agressão física, trauma por acidente de trânsito e trauma por queda, se comparado ao relatório produzido por Teresina; para fins de análise e para traçar um comparativo com mais fidelidade, fez-se a união desses três itens ${ }^{(7)}$.

No entanto, quando comparados os atendimentos produzidos pelo SAMU Teresina e SAMU Florianópolis, constatou-se que os atendimentos por urgência clínica em adulto se assemelham nos quantitativos produzidos, sendo realizado aproximadamente $19 \%$ mais atendimento pelo SAMU Florianópolis, que é o que se espera em decorrência do número maior de habitantes. Em relação às urgências obstétricas, constatou-se uma disparidade nas duas realidades, tendo o SAMU Teresina realizado em média $540 \%$ mais atendimentos desta natureza que o SAMU Florianópolis. Em relação aos traumas e causas externas, aqui consideradas urgências por quedas, acidentes de trânsito e agressão física, pode-se constatar que em Teresina o número de atendimentos ultrapassou em aproximadamente $25 \%$ o de Florianópolis.

Quando analisados os atendimentos referentes a urgências psiquiátricas, verificou-se que o SAMU Teresina realizou, no período em estudo, em média 124\% mais atendimentos dessa natureza. Chama a atenção os índices de falsos chamados que acometem esses serviços: o SAMU Florianópolis foi vítima, em média, de 3.312 falsos chamados/ano, enquanto o SAMU Teresina recebeu em média 330/ano. Dessa forma, pode-se perceber que o número de falsos chamados praticados contra o SAMU Florianópolis foi $854 \%$ maior.

Outro ponto relevante demonstrado é o numero de remoções inter-hospitalares praticados pelo SAMU Teresina, com média de 8.841 ocorrências/ano, enquanto o SAMU Florianópolis realizou no ano de 2010 apenas 28 atendimentos dessa natureza. Observou-se ainda que o SAMU Teresina consolida os óbitos ocorridos antes do socorro e os que ocorrem durante o trans- porte, não sendo visualizada essa particularidade no SAMU Florianópolis. No ano de 2005, em Terezina, o maior quantitativo foi gerado por urgências clínicas em adulto e em urgências obstétricas, com diminuição significativa nos anos subsequentes. No entanto, podese perceber que os traumas por acidentes de trânsito apresentaram uma curva ascendente e ligeira queda no ano de 2008, com posterior elevação nos anos que se seguem. Os demais tipos de solicitações se mantiveram dentro de suas médias, sem elevações ou quedas significativas.

O tempo resposta das equipes do SAMU é o tempo transcorrido desde o momento da recepção do chamado na Central de Regulação médica até a chegada da equipe ao local da ocorrência. $O$ tempo médio de transporte refere-se à média do tempo de transporte do cenário ao hospital de referência. Esses tempos são indicadores de qualidade do serviço ${ }^{(8)}$.

Para o Committee on Trauma of Surgeons, dos Estados Unidos da América, o tempo ideal para o atendimento e realização de procedimentos em pacientes vitimas de traumas é de 20 minutos $^{(9)}$. Dessa forma, foi considerado o tempo resposta como um dos fatores que influenciarão diretamente na qualidade do atendimento e nas chances de sobrevivência e minimização de possibilidades de sequelas, sobretudo das vítimas de agravos violentos e relacionados ao sistema cardiovascular. A regulamentação americana para os serviços médicos de urgência estabelece que $95 \%$ das solicitações em área urbana devem ser atendidas em no máximo, 10 minutos, período estendido para 30 minutos no caso de áreas rurais ${ }^{(8)}$.

Em pesquisa realizada em capitais brasileiras, podese observar que a média do tempo resposta no primeiro semestre de 2005; em Manaus, foi de 2 minutos, em Recife de 15 minutos, no Distrito Federal e em Curitiba, de 10 minutos, e no Rio de Janeiro foi de 9 minutos ${ }^{(10)}$. Constatou-se que o SAMU Teresina está longe do padrão de Manaus, mas próximo dos tempos das demais capitais pesquisadas. Vale ressaltar que esses tempos estão relacionados aos deslocamentos dentro do perímetro urbano dos municípios; dessa forma, constata-se que, em relação ao deslocamento para o local da ocorrência, o SAMU Teresina gasta, na zona urbana, $36 \%$ a mais de tempo que o recomendado pelo Committee on Trauma of Surgeons, dos Estados Unidos da América.

Em relação ao tempo médio gasto no transporte do local da ocorrência até o hospital de referência, em Manaus foi de 5 minutos; em Recife de 10 minutos, no Distrito Federal de 30 minutos, no Rio de Janeiro de 20 
minutos e em Curitiba, de 6 minutos $^{(10)}$. Assim, podese perceber que, em algumas capitais, o tempo médio de deslocamento até o local da ocorrência é menor que o de deslocamento de lá para o hospital, como é o caso de Manaus, Distrito Federal e Rio de Janeiro. No entanto, a realidade de algumas capitais, como Recife e Curitiba, assemelha-se à de Teresina, com diminuição nesse tempo. Acredita-se que, esse evento se deve a fatores como a dificuldade de localização de endereços para a chegada da equipe até as vítimas, distância do local do agravo em relação à localização da unidade móvel de urgência, condições de trafegabilidade, visão do condutor no sentido de optar pelo melhor trajeto do local da ocorrência até o hospital de destino do paciente e descentralização dos hospitais de atendimento. Mesmo havendo essa diminuição, o SAMU Teresina gasta $12 \%$ a mais do tempo recomendado para o transporte da vítima do local do agravo ao hospital no perímetro urbano, ou seja, um minuto e dois segundos.

Após o atendimento no local da ocorrência, é necessário que se referencie o paciente à unidade de saúde mais próxima e com recursos para o atendimento do agravo. É imprescindível que se conheça as características e os recursos disponíveis em cada unidade de saúde, para que se possa dispensar um atendimento hospitalar voltado para as necessidades de cada paciente. A norma técnica n. 11/2006 do Conselho Nacional dos Secretários de Saúde que versa sobre a política nacional de regulação, atribui à central de regulação médica o encargo do referenciamento em todos os níveis de atenção nas redes de prestadores públicos e privados, no intento de assegurar o atendimento a pacientes em condições de urgência e emergência ${ }^{(11)}$.

Vale ressaltar que a política de atuação do SAMU encaminha paciente tanto para a rede pública como para a rede privada, sendo determinante a condição física do paciente, estrutura da unidade de saúde preferida e responsabilização dele, em relação ao ônus que $\mathrm{o}$ atendimento poderá gerar em instituições privadas.

Segundo a Política Nacional de Atenção às Urgências $^{(12)}$, as Unidades não-hospitalares de atendimento às urgências e emergências, denominadas de Unidades de Pronto Atendimento (UPA) devem funcionar 24 horas por dia, habilitadas a prestar assistência correspondente ao primeiro nível da média complexidade. É fundamental que as unidades tenham uma retaguarda adequada para o referenciamento daqueles pacientes que, uma vez acolhidos, avaliados e tratados nesse primeiro nível de assistência, necessitem de cuidados disponíveis em serviços de outros níveis de complexidade. No entanto, a política praticada pelo SAMU se baseia nos Planos de Atenção Integral às Urgências, que deixam claro o compromisso de atuação de cada unidade formadora da rede com relação à implantação da política denominada "vaga zero" nas portas de urgência, conforme disposto na Portaria n. 2.048/02(13).

Fica explicita, nos quantitativos obtidos pelo SAMU Teresina, uma preocupação de não superlotar os hospitais de referência do estado. Dessa forma, há uma evidente preocupação em diluir os atendimentos em hospitais de menor complexidade, com o nível de resolutibilidade assegurado.

\section{CONCLUSÃO}

A rede nacional SAMU 192 se configura em uma das vertentes que atua visando à regulação e ao atendimento das urgências e emergências pré-hospitalares. Isso confere um atendimento presencial ou por telemedicina a todas as chamadas, que busca levar a população uma resposta apropriada. Desta forma procura assegurar a recepção dos pacientes, além de proporcionar atendimento contínuo nas viaturas, até o ingresso do paciente na rede hospitalar.

Os atendimentos de urgências clínicas em adultos, seguidas por urgências obstétricas e atendimento a vítimas de acidentes de trânsito reflete a situação de municípios cuja população atendida é constituída, em sua maioria, de pessoas de baixo poder aquisitivo, e que necessita recorrer a esse serviço para receber atendimento médico, e de adolescentes e adultos jovens das diversas classes sociais, vítimas de acidentes de trânsito e de atos de violência, o que remete à realidade de uma das capitais brasileiras com maior índice de agravos por causas externas.

Percebe-se a necessidade de diminuir o tempo de deslocamento no intuito de prestar atendimento ágil à população, as condições de tráfego e a educação no trânsito impactam sobremaneira no tempo resposta e influenciam no tempo de chegada da equipe ao local da ocorrência e daí ao serviço de referência, salientandose a capacidade operacional constantemente extrapolada do hospital público de referência, o que, para o SAMU, implica morosidade na entrega do paciente e resgate de material permanente das viaturas, indispensável ao atendimento a outras vitimas de trauma.

\section{REFERÊNCIAS}

1. Maciak I, Sandri J, Spier F. Humanização da assistência de enfermagem em uma unidade de emergência: percepção do usuário. Cogitare enferm. 2009;14(1):127-35. 
2. Stumm E, Ribeiro G, Kirchner R, Loro M, Rosanelli C. Avaliação da saúde e qualidade de vida: profissionais de um samu. Cogitare enferm. 2009;14(4):620-27.

3. Ministério da Saúde(BR). Manual de regulação médica das urgências. Brasília: Ministério da Saúde, 2006.

4. Uchimura KY, Bosi MLM. Qualidade e subjetividade na Avaliação de Programas em Saúde e Serviços. Cad. Saúde Publica. 2002;18(6):1561-9.

5. Donabedian A. La dimensión internacional de la evaluación y garantía de la calidad. Salud publica de México 1990;32:113-7.

6. Contandriopoulos AP, Champagne F, Denis JL, Pineault R. A Avaliação na área da saúde: conceitos e métodos. In: Harzt ZMA, organizadora. Avaliação em saúde: dos modelos conceituais à prática na análise da implantação de programas. Rio de Janeiro: FIOCRUZ; 1997. p. 29-47.

7. Secretaria de Estado da Saúde (BR). Relatório ano 2010. SAMU 192. Santa Catarina: Superintendência de Serviços Especializados e Regulação. Gerência Estadual do SAMU/192, 2011.

8. Macedo, CRB. Qualidade nos serviços de urgência: alguns indicadores. Seminário de Política Nacional Integral as Urgências. [acesso em 24 out 2010]. Disponível: http://www.saude.ba.gov.br.

9. Whitaker IY, Gutiérrez MGR de, Koizumi MS. Gravidade do trauma avaliada na fase pré-hospitalar. Rev. Assoc. Med. Bras. 1998;44(2):111-9.

10. Minayo MCS, Deslandes SF. Análise da implantação do sistema de atendimento pré-hospitalar móvel em cinco capitais brasileiras. Cad. Saúde Pública. 2008; 24(8):1877-86.

11. Ministério da Saúde (BR). Conselho Nacional de Secretários de Saúde. Norma Técnica n.11. Versa sobre a política nacional de regulação. Brasília: Ministério da Saúde 2006.

12. Ministério da Saúde (BR). Política nacional de atenção às urgências. $3^{\mathrm{a}}$ ed. ampliada. Brasília: Ministério da Saúde, 2006.

13. Ministério da Saúde (BR). Portaria n. 2048, de 05 de Novembro de 2002. Aprova o Regulamento Técnico dos Sistemas Estaduais de Urgência e Emergência. Brasília; 2002. 